\title{
Tipos de mantenimiento en las plantas compresoras de gas de la industria petrolera venezolana Distrito-Lagunillas
}

\section{Types of maintenance in the gas compressor plants of the Venezuelan oil industry District-Lagunillas}

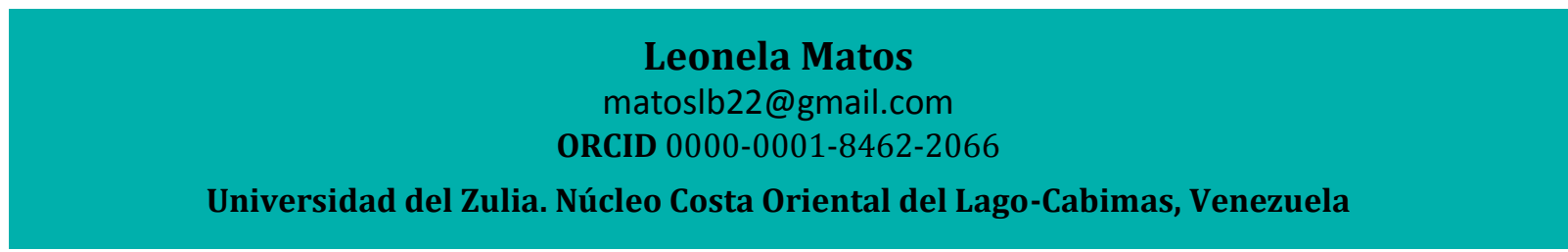

Artículo recibido septiembre 2019 | Arbitrado en octubre 2019 | Publicado en enero 2020

\section{RESUMEN}

En el presente trabajo de investigación se describió los tipos de mantenimiento realizados en plantas compresoras de gas en la industria petrolera venezolana, Distrito Lagunillas. La metodología se consideró de tipo descriptiva, bajo un diseño no experimental, de campo y transeccional. La población quedó conformada por las 3 plantas compresoras de gas de la industria petrolera venezolana en el Distrito Lagunillas. Como técnica de recolección de datos se empleó la encuesta mediante un cuestionario estructurado por 15 ítems con escala dicotómica. Para alcanzar la validez del mismo fue sometido al juicio de expertos, y se utilizó el coeficiente Kuder y Richardson para el cálculo de su confiabilidad, obteniéndose 0,91. El análisis de los datos se realizó mediante la estadística descriptiva, a través de las frecuencias relativas. Se concluye que estas plantas otorgan alta presencia a los tipos de mantenimiento que llevan a cabo: predictivo, preventivo, programado, correctivo y proactivo.

Palabras clave: Correctivo; predictivo; preventivo; proactivo; programado; tipos de mantenimiento

\section{ABSTRACT}

In this research paper, describe the types of maintenance performed in gas compressor plants in the Venezuelan oil industry, Lagunillas District. The methodology is considered descriptive, under a non-experimental, field and transectional design. The population was made up of the 3 gas compressor plants of the Venezuelan oil industry in the Lagunillas District. As a data collection technique, the survey was used using a questionnaire structured by 15 items with a dichotomous scale. To reach its validity, it was at some point the experts' judgment, and the Kuder and Richardson coefficient was considered to calculate its reliability, obtaining 0.91 . Data analysis was carried out using descriptive statistics, using specific frequencies. It is concluded that these plants give high presence to the types of maintenance they carry out: predictive, preventive, scheduled, corrective and proactive.

Key words: Corrective, predictive, preventive, proactive, scheduled, types of maintenance 


\section{INTRODUCCIÓN}

En el contexto mundial las empresas del ámbito petrolero incluyen procesos globales de exploración, extracción, refinación, transporte, mercadotecnia de los productos del petróleo. Para ello, sus formas de mantenimiento han venido evolucionando, centrados a la preservación de activos productivos, alargando la vida útil $\mathrm{y}$ económica de sus plantas-máquinasherramientas, reduciendo su desgaste físico en un corto plazo.

Cabe destacar, que sus procesos industriales tienen asociados altos riesgos, que si no se eliminan pueden generar consecuencias negativas incuantificables, pudiendo generar impactos a las personas, instalaciones, ambiente, producción, así como la calidad de vida. Es así como la prevención, y las condiciones de seguridad, pueden minimizar en gran medida cualquier evento que se presente, ya sea por fallas; falta $y / o$ deficiente mantenimiento, procedimientos operativos inadecuados, falta de ellos, o su inobservancia, mala calidad de materiales de mantenimiento, mano de obra inadecuada o simplemente la aparición de un evento impredecible.

Sobre esta base, para Venahin (2008) el mantenimiento es la herramienta de toda empresa para conservar operable, con el debido grado de eficiencia y eficacia, su activo fijo, engloba al conjunto de actividades necesarias para mantener una instalación o equipo en funcionamiento o restablecer el mismo en condiciones predeterminadas, el cual incide en la cantidad y calidad de la producción, evitando la paralización de los procesos, y evitando que incida en el proceso productivo de la organización.

De acuerdo a esto, se vislumbra la necesidad de organizar adecuadamente el servicio, introduciendo programas $\mathrm{y}$ controles correctivos para optimizar la disponibilidad de los equipos, considerándolo como un servicio que agrupa actividades cuya ejecución permiten alcanzar mayor confiabilidad en los equipos, máquinas, plantas, entre otros.

Ahora bien, para Segura (2005), la gestión de mantenimiento es un proceso sistemático donde se deben planear acciones ayudados por procedimientos que lleven una secuencia lógica a fin de conseguir confiabilidad y disponibilidad de los objetos a mantener. En este aspecto, existe una amplia concordancia entre diversos autores, tales como Venahin (2008) y Perdomo (2007), quienes afirman que la ingeniería está recibiendo cada vez más atención, especialmente debido a la necesidad de obtener de los equipamientos, de alto costo, una alta productividad, como también, mediante un efectivo mantenimiento, influir fuertemente en el diferencial competitivo de su producto.

En este orden, para Perdomo (2007) la forma correcta de direccionar las necesidades para una función de mantenimiento efectiva dentro de la organización, es teniendo una visión holística de la función. Otro punto en destaque es el alcance de un real mejoramiento, lo cual requiere integrar completamente al mantenimiento en el sistema de negocios de la empresa, especialmente usando tecnologías de información y formulando una concepción con bases teóricas comprobadas.

Asimismo, dentro de las empresas operativas, se necesitan ciertas reglas las cuales deben estar plasmadas para su efectivo funcionamiento, seguridad, operatividad, además, deben ser aceptadas y analizadas. Actualmente para la sociedad, es 
de suma importancia que las organizaciones no sólo posean sino que brinden seguridad y ésta sea proyectada ante sus distintos públicos.

En este marco de referencia, en Venezuela, como ámbito territorial latinoamericano, el mantenimiento surgió por la necesidad de disminuir las correcciones así como todo lo que representaba, reduciendo la reparación mediante inspecciones periódicas y la renovación en los elementos dañados, por lo que la misma debía ser constante hasta el logro de los objetivos.

En específico, la empresa estatal Petróleos de Venezuela, S. A. (PDVSA) en la Costa Oriental del Lago, según la observación directa de la investigadora por ser parte activa de este sector, ha venido desarrollando sus actividades de explotación, producción, refinación, mercado y transporte del gas venezolano, basándose en el mejoramiento continuo de todos los procesos, para garantizar la satisfacción de los clientes, siendo pertinente denotar la situación presentada en las plantas compresoras de gas.

De acuerdo con una entrevista preliminar, realizada al director de dicho ramo empresarial petrolero, el mantenimiento de éstas no se encuentra organizado para ejecutarse según la periodicidad, elaboración y desarrollo; además, no se reconoce la importancia operativa y económica que este ocasiona. Por otra parte, asegura el entrevistado, los trabajadores de estas empresas no conocen cuales son las ventajas y desventajas de mantener las plantas en buen estado de uso y producción, lo cual es una amenaza de orden interno. Aunado a lo expuesto, se vienen percibiendo una serie de carencias específicas en las plantas compresoras de gas, una de ellas es la falta de adiestramiento del personal en su manipulación, así como en su mantenimiento, lo que podría traer graves consecuencias a futuro, afectando la producción y los objetivos.

Es decir, al ejecutar cualquier operación en las plantas compresoras de gas podrían ocurrir accidentes. A ello se suma la interrupción en la producción, lo cual significaría un retraso económico para el país y pudiera presentarse insatisfacción de los clientes o empresas que requieren el producto final. Todo esto traería consigo el deterioro en los sistemas de producción en las plantas compresoras de gas, lo cual afectaría de manera considerable la capacidad productiva y su competitividad en el mercado gasífero. Es importante señalar que si no se realizan los correctivos pertinentes la empresa podría padecer consecuencias graves en las plantas compresoras, debido a que éstas son la base fundamental para la producción gasífera, aportando un papel relevante ante el desarrollo y satisfacción de sus clientes, así como a la economía venezolana.

Es este sentido, para lograr un desarrollo efectivo de sus operaciones se deben unificar criterios de mantenimiento de las plantas compresoras de gas, por lo cual es pertinente plantear acciones $\mathrm{y}$ procedimientos que lleven una secuencia lógica de actividades, a fin de conseguir confiabilidad y disponibilidad de los objetos a mantener, tal y como lo afirma Segura (2005).

Ahora bien, con el fin de que estas plantas den respuesta a las necesidades industriales y a las diversas variables existentes en el área petrolera, se deben asumir acciones sistemáticas encargadas de organizar y de administrar los recursos, de manera tal que se pueda concretar todo el 
trabajo requerido por un proyecto dentro del tiempo y del presupuesto disponible para el mantenimiento. Debido a la situación antes mencionada, se hace relevante conocer como estas plantas compresoras de gas están llevando a cabo sus operaciones de mantenimiento, siendo pertinente, a juicio de la investigadora, describir los tipos de mantenimiento aplicados en las plantas compresoras de gas en la industria petrolera venezolana, Distrito Lagunillas.

\section{Gestión de mantenimiento}

Duffuaa y otros (2010) definen la gestión de mantenimiento como la manera en la cual se especifican y controlan las prácticas requeridas para cumplir con los planes organizacionales, normalmente se basan en el círculo de calidad (planificación, hacer, verificar y actuar). De esta manera, la gestión de mantenimiento es un mecanismo muy valioso para asegurar que los principios de planificación total del ciclo de vida, gestión de riesgo, costo/beneficio, enfoque al cliente, sustentabilidad, entre otros, sea realmente implementados dentro del trabajo diario de desarrollo de proyectos de capital, operaciones, mantenimiento, $\mathrm{u}$ otros.

Desde la perspectiva de Zambrano y Leal (2006), la gestión de mantenimiento es un proceso sistémico donde a través de una serie de medidas organizativas se pueden planear las acciones de las actividades de mantenimiento por medio de procedimientos que lleven un orden $o$ secuencia lógica de esta función a fin de conseguir un constante y adecuado desempeño de los equipos pertenecientes al sistema productivo, esto con la finalidad de identificar los pasos a seguir y prever las posibles desviaciones que se puedan presentar durante el desarrollo de estas actividades de mantenimiento.
En esta misma orientación, Rodríguez (2008) define la gestión del mantenimiento como el conjunto de actividades de diseño, planificación y control que tienen por objeto minimizar los costos asociados al mal funcionamiento de los equipos; agrega este autor que además de las actividades típicas de mantenimiento, debe incluirse la formación del personal, su capacitación y adiestramiento deben ser parte clave de esta gestión.

A juicio de la investigadora, la gestión de mantenimiento se basa en actuar sobre todos los aspectos de importancia para el óptimo funcionamiento de la empresa. El departamento de mantenimiento no debe limitarse solamente a la reparación de las instalaciones, sino también debe pilotear los costos de mantenimiento, recursos humanos y almacenes a fin de desarrollar una excelente gestión.

\section{Tipos de mantenimiento}

Para Duffuaa y otros (2010) los tipos de mantenimiento han evolucionado con el tiempo, actualmente existen variados sistemas que pueden presentarse en las instalaciones de operación, que exigen diferentes modos de mantenimiento para preservar la continuidad de los procesos productivos y sustentar la rentabilidad operacional, entre los cuales se encuentran: predictivo, preventivo, programado, correctivo y proactivo.

De acuerdo a lo anterior, se puede señalar que los tipos de mantenimiento permiten a la organización conocer los requerimientos de cada máquina y equipo, para ello, es clave realizarlo dependiendo de las necesidades de la organización, logrando proporcionarle el servicio necesario para que la las actividades se realicen de acuerdo a lo establecido. 
En este contexto, para Nava (2010), cuando se habla de tipos de mantenimiento, es más correcto hablar de tipos de tareas de mantenimiento, y en este sentido, existen diferentes clasificaciones de las tareas según distintos criterios. La clasificación más extendida se refiere a la naturaleza de las tareas, así, se han distinguido seis tipos de tareas de mantenimiento, que se diferencian entre sí por el carácter de las tareas que incluyen: correctivo, preventivo, conductivo, predictivo, cero horas, y modificativo.

Ahora bien, Jiménez y Milano (2006) son más específicos en este aspecto, y afirman que los tipos de mantenimiento se deben establecer con base en análisis económicos de criticidad y de riesgo de los equipos. Consideran, que para la buena ejecución y desempeño de las políticas de mantenimiento, se requiere una complementación de las diferentes categorías, buscando de esta forma una adecuada centralización de los distintos enfoques con sus características propias. De acuerdo a esta caracterización, se pueden realizar: mantenimiento correctivo, preventivo y predictivo.

En este orden de ideas, García (2009) afirma que la mayor parte de lo que se ha escrito hasta ahora sobre tipos de mantenimiento, hacen referencia, básicamente, a cuatro tipos: predictivo, preventivo, correctivo y proactivo; a diferencia de los autores anteriores García agrega el tipo proactivo. Así, afirma García, en una organización estos cuatro tipos no son excluyentes, sino cuando se plantea la estrategia a seguir, normalmente la respuesta es una combinación de los tipos de mantenimiento anteriores.

A su vez, considera este autor, que los 4 tipos de mantenimiento señalados permiten coordinar, dirigir, organizar los recursos materiales, humanos y flujos de información destinados al correcto funcionamiento, reparación, así como prolongación de la vida de los equipos disponibles, para que, además, de lograr eficacia en las labores propias de mantenimiento se consiga una contención del gasto y la optimización de costos.

Con base en la exposición antes realizada, sobre los tipos de mantenimiento, para los fines de la presente investigación se consideró como base el criterio expuesto por García (2009) en relación a los tipos de mantenimiento, quien considera en conjunto 4 tipos: predictivo, preventivo, correctivo y proactivo; aunque también se agrega a estos básicos el programado, propuesto por Duffuaa y otros (2010). Esto obedece al criterio de la investigadora, dado que forma parte del sector bajo estudio, considerando que serían los de mayor pertinencia y aplicabilidad dentro del sector. Seguidamente serán objeto de análisis y se exponen detalladamente a continuación.

\section{Predictivo}

Para Duffuaa y otros (2010) el mantenimiento predictivo consiste en determinar en todo instante la condición técnica (mecánica y eléctrica) real de la máquina examinada, mientras ésta se encuentre en pleno funcionamiento, para ello se hace uso de un programa sistemático de mediciones de parámetros del equipo. El sustento tecnológico de este mantenimiento consiste en aplicaciones de algoritmos matemáticos agregados a las operaciones de diagnóstico, que juntos pueden brindar información referente a las condiciones del equipo. Tiene como objetivo disminuir las paradas por mantenimiento preventivos, $\mathrm{y}$ así minimizar los costos por mantenimiento y por no producción. 
De acuerdo a Nava (2010), este tipo de mantenimiento se basa en predecir la falla antes de que ésta se produzca. Se trata de adelantarse al momento en que el equipo deja de trabajar en sus condiciones óptimas. Para lograrlo se utilizan herramientas y técnicas de monitores de parámetros físicos. Involucra actividades que se desarrollan para detectar cualquier anomalía en equipos en funcionamiento, aplicando la tecnología para la interpretación de datos obtenidos a través de un diagnóstico, con instrumentos colocados en diferentes partes del equipo o bien sea por toma de muestras.

Este tipo de mantenimiento no es dependiente de la característica de la falla, y es el más efectivo cuando el modo de falla es detectable por monitoreo de las condiciones de operación. Se lleva a cabo en forma calendario y no implica poner fuera de operación los equipos. Entre las técnicas usadas están las inspecciones, el chequeo de condiciones y el análisis de tendencias.

Afirman Zambrano y Leal (2007), que es el mantenimiento planificado y programado basándose en análisis técnicos y en la condición del equipo, antes de ocurrir una falla, sin detener el funcionamiento normal del equipo, para determinar la expectativa de vida de los componentes y reemplazarlos en tiempo óptimo. Se basa primordialmente en diseños electrónicos, son elementos adheridos a los sistemas.

Básicamente, a criterio de la investigadora, este tipo de mantenimiento consiste en reemplazar o reparar partes, piezas, componentes o elementos justo antes que empiecen a fallar o a dañarse. En el programa de este tipo de mantenimiento se analizan las condiciones del equipo mientras este se encuentra funcionando $o$ en operación, permitiendo de esta manera ajustar las operaciones y su periodicidad a un máximo de eficiencia. Esto es siempre menos costoso y confiable que el intervalo de mantenimiento preventivo, basado en factores como las horas máquina o alguna fecha prefijada. Por ello, se deben realizar controles aleatorios o basados en la experiencia de los operadores de los equipos y gente de mantenimiento, lo cual es un soporte a la hora de evitar daños mayores o que se produzcan por efecto de las paradas forzadas.

\section{Preventivo}

Para Duffuaa y otros (2010) el mantenimiento preventivo, también llamado planificado, se da antes de que ocurra la falla o avería y se efectúa en condiciones controladas, sin la existencia de algún error en el sistema. Se realiza a razón de la experiencia, pericia del personal a cargo, los cuales son los encargados de determinar el momento necesario para llevar a cabo dicho procedimiento; también se puede estipular el momento adecuado a través de manuales técnicos.

Estos autores lo definen como un proceso planificado, estructurado y controlado de tareas de mantenimiento a realizar dentro de las recurrencias establecidas, las mismas que generalmente son definidas por los fabricantes, y a falta de éstas se puede recurrir a las mejores prácticas del mercado de este tipo de servicios.

Presenta las siguientes características, según Torres (2006):

- Se realiza en un momento en que no se está produciendo, por lo que se aprovechan las horas ociosas de la planta. 
- Sigue un programa previamente elaborado, donde se detalla el procedimiento a seguir, actividades a realizar, a fin de tener las herramientas y repuestos necesarios.

- Cuenta con una fecha programada, además de un tiempo de inicio y de terminación preestablecido y aprobado por la directiva de la empresa.

- Está destinado a un área en particular, y a ciertos equipos específicamente. Aunque también se puede llevar un mantenimiento general de los componentes de la planta.

- Permite a la empresa contar con un historial de todos los equipos, además brinda la posibilidad de actualizar la información técnica de los equipos.

- Permite contar con un presupuesto aprobado por la directiva.

- Se aplica y se programa con el objeto de inspeccionar, reparar y cambiar parte de un equipo antes de ocurra la falla, eliminando o reduciendo los gastos.

Es importante señalar que según este concepto la lubricación, la limpieza, el ajuste de los equipos cuando trabajan, limpieza de la superficie, no son tareas del mantenimiento rutinario $\mathrm{y}$ es necesario realizarlo cuando corresponda mientras que se realiza el mantenimiento preventivo, esta teoría tiene que ser planteada y controlada.

Por su parte, Salih (2010) define el mantenimiento preventivo como una serie de tareas planeadas previamente que se llevan a cabo para contrarrestar las causas conocidas de fallas potenciales de las funciones para las que fue creado un activo. Puede planearse y programarse con base en el tiempo, el uso o la condición del tiempo. Mientras que para Newbrough (2010), el mantenimiento preventivo se define como el conjunto de acciones periódicas necesarias para conservar un equipo en buen estado. Independientemente del comportamiento o de la aparición de una falla se le dará a un equipo, y variará de acuerdo a su utilización y la necesidad de la producción del equipo.

Por lo antes planteado, el mantenimiento preventivo es el que utiliza todos los medios disponibles, incluso los estadísticos, para determinar la frecuencia de las inspecciones, revisiones, sustitución de piezas claves, probabilidad de aparición de averías, vida útil, u otras. A efectos de la investigadora, este tipo de mantenimiento surge de la necesidad de rebajar el correctivo y todo lo que representa, como reducir la reparación mediante una rutina de inspecciones periódicas y la renovación de los elementos dañados. Así entonces, consiste en programar revisiones de equipos, apoyándose en la experiencia y los históricos obtenidos de las máquinas y equipos.

\section{Programado}

Según González (2005), el mantenimiento programado es el que se efectúa a intervalos predeterminados de tiempo, número de operaciones, recorridos, entre otros. Consiste en establecer controles para detectar fallos potenciales, servicios programados y revisiones planificadas, más la sustitución de las piezas averiadas o con tendencia a la avería. Equivale al término mantenimiento rutinario, planificado o sistemático. Presta mucha atención a las recomendaciones del fabricante $\mathrm{y}$ otras personas conocedoras del tema, para obtener un cronograma de aplicación.

Por otro lado, de acuerdo a la Norma Covenin 3049-93 (1993), el mantenimiento programado toma como basamento las instrucciones técnicas recomendadas por los 
fabricantes, constructores, diseñadores, usuarios y experiencias, para obtener ciclos de revisión y/o sustituciones para los elementos más importantes de un sistema productivo a objeto de determinar la carga de trabajo que es necesario programar. $\mathrm{Su}$ frecuencia de ejecución cubre desde quincenal hasta generalmente periodos de un año. Es ejecutado por la cuadrillas de la organización que se dirigen al sitio para realizar las labores incorporadas en un calendario anual.

En este mismo orden de ideas, Trashorras (2006) explica que este tipo es el conjunto de acciones de mantenimiento que se aplican de forma programada en el tiempo, bien por horas de servicio, por número de ciclos de trabajo o, simplemente, por un tiempo natural transcurrido. Puede comprender acciones como sustitución de componentes, desmantelamiento y montaje, para reconocer componentes ocultas, sustitución de líquidos u otros fluidos, engrases, entre otros. Ahora bien, Martínez (2007) afirma que en este tipo de mantenimiento los métodos de programación son estadísticos, y se basan en las experiencias del fabricante de los equipos y componentes, para determinar la frecuencia con que se ha de aplicar el mantenimiento.

Tomando en consideración las definiciones anteriores, a juicio de la investigadora, se puede decir que el mantenimiento programado, es el conjunto de distintas actividades programadas con el fin de llevar a cabo un desempeño productivo para la máquina. El objetivo de estas actividades es que la máquina no tenga ningún tipo de averías, defectos o despilfarros. Por lo cual, este surgirá como el resultado de la dedicación del departamento de mantenimiento, teniendo como objetivo ajustar la programación del equipo para desarrollar las tareas en el momento menos perjudicial para la producción.

\section{Correctivo}

Plantean Duffuaa y col. (2010) que el tipo de mantenimiento correctivo, también denominado mantenimiento reactivo, tiene lugar luego que ocurre la falla o avería, es decir, solo actuará cuando se presenta un error en el sistema. En este caso si no se produce ninguna falla el mantenimiento será nulo, por lo que se tendrá que esperar hasta que se presente el desperfecto para recién tomar medidas de corrección de errores, lo cual trae consigo las siguientes consecuencias, según los autores citados:

- Paradas no previstas en el proceso productivo, disminuyendo las horas operativas.

- Afecta las cadenas productivas, es decir, que los ciclos productivos posteriores se verán parados a la espera de la corrección de la etapa anterior.

- Presenta costos por reparación y repuestos no presupuestados, por lo que se dará el caso que por falta de recursos económicos no se podrán comprar los repuestos en el momento deseado.

- El tiempo que estará el sistema fuera de operación no es predecible.

- Consiste en corregir una falla que se origina en un equipo.

Por tanto, la acción de carácter puntual a raíz del uso, agotamiento de la vida útil $u$ otros factores externos, de componentes, partes, piezas, materiales $\mathrm{y}$, en general, de elementos que constituyen la infraestructura o planta, permitiendo su recuperación, restauración o renovación, no agrega valor al establecimiento. 
En este marco de referencia, para Nava (2010) al tipo correctivo también se le conoce como mantenimiento reactivo, es aquel trabajo que involucra una cantidad determinada de tareas de reparación no programadas con el objetivo de restaurar la función de un activo una vez producido un paro imprevisto (parada forzada). En este contexto, afirman Zambrano y Leal (2007), que las causas que pueden originar un paro imprevisto se deben a desperfectos no detectados durante las inspecciones predictivas, errores operacionales, ausencia de tareas de mantenimiento (reparaciones), sobre uso o utilización de los equipos fuera de las condiciones normales de operatividad del diseño, y a problemas de fabricación de partes o piezas de equipos.

Ahora bien, para García (2009), este tipo de mantenimiento puede presentar algunas ventajas como también desventajas. Entre las ventajas principales de esta estrategia señala: si el equipo está preparado, la intervención en el fallo es rápida y la reposición será con el mínimo tiempo; no se necesita una infraestructura excesiva, un grupo de operarios competentes será suficiente, por lo tanto el costo de mano de obra será mínimo, será más prioritaria la experiencia y la pericia de los operarios que la capacidad de análisis o de estudio del tipo de problema que se produzca; y es rentable en equipos que no intervienen de manera instantánea en la producción.

Mientras que las desventajas más predominantes serían: se producen paradas con daños imprevisibles en la producción que afectan a la planificación de manera incontrolada; se suele producir una baja calidad en las reparaciones debido a la rapidez en la intervención, y a la prioridad de reponer antes que reparar definitivamente, por lo que produce un hábito a trabajar defectuosamente, sensación de insatisfacción e impotencia, ya que este tipo de intervenciones a menudo generan otras al cabo del tiempo por mala reparación por lo tanto será muy difícil romper con esta inercia.

En síntesis, para la investigadora, la principal función de una gestión adecuada del mantenimiento debe consistir en rebajar el correctivo hasta el nivel óptimo de rentabilidad para la empresa. No obstante, el correctivo no se puede eliminar en su totalidad, por lo tanto, una gestión correcta extraerá conclusiones de cada parada e intentará realizar la reparación de manera definitiva, ya sea en el mismo momento o programando un paro, para que esa falla no se repita. Por lo cual es importante tener en cuenta en el análisis de la política de mantenimiento a implementar, que en algunas máquinas o instalaciones el correctivo sería el sistema más rentable.

\section{Proactivo}

Para Torres (2006), el mantenimiento proactivo comprende actividades que permiten aumentar la vida útil del equipo a través de un control de origen de la falla, utilizando las herramientas de un programa de monitoreo de condición, el cual se enfoca a las causas y no a los efectos de las mismas, a diferencia del mantenimiento predictivo. También toma en cuenta las máquinas criticas de un sistema o instalación, análisis de modos de fallas, consecuencias, síntomas, efectos, y determina los objetivos de control para cada una de ellas, además de los tipos de análisis que se efectuarán y las medidas para volver a los valores establecidos. Su objetivo es extender la vida de la maquinaria, identificando la causa raíz que genera el desgaste, para mantener los 
parámetros de causa de falla dentro de límites aceptables.

Según Nava (2010), este tipo de mantenimiento es utilizado para mejorar el rendimiento de cualquier organización, considerando el estudio de probabilidad de falla, y disminuyendo el riesgo de aparición de la misma. Todo esto debe cumplirse para tratar de obtener costos totales mínimos de operación, operando en buenas condiciones durante un porcentaje de tiempo óptimo.

Ahora bien, García (2009) señala al tipo proactivo como una gran cantidad de técnicas para alargar la disponibilidad de las máquinas. Considera que la idea principal de un mantenimiento proactivo es el análisis de las causas fundamentales de las fallas, así estas causas se pueden remediar y los mecanismos de falla que se puedan eliminar gradualmente, mejoraran la operación de las máquinas. Visto así, el mantenimiento proactivo no se encarga de corregir los errores o defectos de las máquinas si no en lo que los causa. Lo que implicaría uno de los mantenimientos más baratos.

Por su parte, Altmann (2005) considera que en este mantenimiento se utilizan técnicas especializadas para monitorear la condición de los equipos, basándose en el análisis de aceite para establecer el control de los parámetros de causa-falla. Asimismo, se establece una técnica de detección temprana, monitoreando el cambio en la tendencia de los parámetros considerados como causa-falla, para tomar acciones que permitan al equipo regresar a las condiciones establecidas y desempeñarse adecuadamente.

De acuerdo a lo planteado, se puede señalar que este tipo de mantenimiento permite tener un control de las máquinas y equipos, fortaleciendo los procesos mediante análisis de modos de fallas, criticidad, costos, extendiendo así la vida útil de los equipos de la organización. Para la investigadora, es importante considerar que la productividad aumentará en la medida en que las fallas de las máquinas disminuyan de una forma sustentable en el tiempo. Para lograrlo, resulta indispensable contar con la estrategia de mantenimiento más apropiada y con personal capacitado tanto en el uso de las técnicas de análisis como en el diagnóstico de fallas implementadas, con conocimiento suficiente sobre las características de diseño y funcionamiento de las máquinas.

A manera de resumen de esta dimensión, en este punto del trabajo se mencionaron los tipos de mantenimiento utilizados con mayor frecuencia hoy en día, y las técnicas más apropiadas para aplicarlos, entre las que se destaca el análisis de fallas, ilustrando su alcance así como la necesidad de usarla con el fin de llegar a un diagnóstico acertado. Se deduce, que diagnosticado y solucionado los problemas, la vida de las máquinas y su producción aumentará, por tanto, los costos de mantenimiento disminuirán. Cabe resaltar, que la elección del tipo de mantenimiento dependerá en gran medida de las estrategias que se hayan seleccionado en la planificación del mantenimiento.

\section{MÉTODO}

La metodología se consideró de tipo descriptiva, bajo un diseño no experimental, de campo y transeccional. La población quedó conformada por las 3 plantas compresoras de gas de la industria petrolera venezolana en el Distrito Lagunillas, cuyos informantes fueron 28 personas, compuestas por 4 gerentes, 3 líderes y 21 supervisores pertenecientes a las mismas. 
Como técnica de recolección de datos se empleó la encuesta mediante la aplicación de un cuestionario estructurado por 15 ítems con escala dicotómica. Para alcanzar la validez del mismo fue sometido al juicio de cinco expertos, y se utilizó el coeficiente Kuder y Richardson para el cálculo de su confiabilidad, donde se obtuvo como valor de confiabilidad total del instrumento 0,91, al incluir todos los ítems.

El análisis de los datos se realizó mediante la estadística descriptiva, a través del estudio de las frecuencias relativas por medio de la aplicación del baremo de interpretación correspondiente, tal como se muestra en el cuadro 1.

Cuadro 1. Baremo de interpretación de la frecuencia

\begin{tabular}{cc}
\hline \multicolumn{2}{c}{ RANGO PARA FRECUENCIA RELATIVA } \\
\hline $\mathrm{SI} \geq 70 \%$ & Alta presencia \\
$40 \% \leq \mathrm{SI}<70 \%$ & Mediana presencia \\
$\mathrm{SI}<40 \%$ & Baja presencia \\
\hline
\end{tabular}

\section{RESULTADOS}

Para la dimensión tipos de mantenimiento, cuyos resultados se concentran en la tabla 1, se observa cómo el $87,62 \%$, en promedio, de los encuestados consideraron la opción afirmativa, indicando alta presencia de los tipos de mantenimiento aplicados en las plantas compresoras de gas en la industria petrolera venezolana, Distrito Lagunillas.

Tabla 1. Tipos de mantenimiento

\begin{tabular}{|c|c|c|c|}
\hline Indicador: Predictivo & Si (\%) & No (\%) & Categoría \\
\hline $\begin{array}{l}\text { 1. ¿Se evalúan las máquinas antes de iniciar una actividad } \\
\text { vinculada en las planteas compresoras de gas? } \\
\text { 2. ¿Cuenta la empresa con un historial de todos los } \\
\text { equipos que requieren mantenimiento? } \\
\text { 3. ¿Se analizan las condiciones de los equipos mientras se } \\
\text { encuentren en funcionamiento? }\end{array}$ & $\begin{array}{l}28 \\
100 \% \\
28 \\
100 \% \\
28 \\
100 \%\end{array}$ & $\begin{array}{c}0 \\
0 \% \\
0 \\
0 \% \\
0 \\
0 \%\end{array}$ & $\begin{array}{c}100 \% \\
\text { Alta } \\
\text { presencia }\end{array}$ \\
\hline \multicolumn{4}{|l|}{ Indicador: Preventivo } \\
\hline $\begin{array}{l}\text { 4. ¿Se ejecutan actividades para determinar la condición } \\
\text { real de las máquinas examinadas? } \\
\text { 5. ¿Se analizan las condiciones de los equipos mientras se } \\
\text { encuentran funcionando? } \\
\text { 6. ¿Cuenta la empresa con un historial del mantenimiento } \\
\text { realizado a todos los equipos? }\end{array}$ & $\begin{array}{l}28 \\
100 \% \\
28 \\
100 \% \\
25 \\
89,3 \%\end{array}$ & $\begin{array}{c}0 \\
0 \% \\
0 \\
0 \% \\
3 \\
10,7 \%\end{array}$ & $\begin{array}{c}96,4 \% \\
\text { Alta } \\
\text { presencia }\end{array}$ \\
\hline \multicolumn{4}{|l|}{ Indicador: Programado } \\
\hline $\begin{array}{l}\text { 7. ¿Establece controles destinados a detectar fallas } \\
\text { potenciales? } \\
\text { 8. ¿Se ejecuta el mantenimiento programado por } \\
\text { cuadrillas siguiendo una planificación? } \\
\text { 9. ¿Se programan las actividades para llevar a cabo un } \\
\text { desempeño productivo efectivo? }\end{array}$ & $\begin{array}{l}22 \\
78,6 \% \\
22 \\
78,6 \% \\
22 \\
78,6 \%\end{array}$ & $\begin{array}{l}6 \\
21,4 \\
6 \\
21,4 \\
6 \\
21,4\end{array}$ & $\begin{array}{c}78,6 \\
\text { Alta } \\
\text { presencia }\end{array}$ \\
\hline
\end{tabular}




\begin{tabular}{|c|c|c|c|}
\hline Indicador: Correctivo & Si (\%) & No (\%) & Categoría \\
\hline $\begin{array}{l}\text { 1. ¿Se realizan actividades correctivas cuando ocurre una } \\
\text { falla? }\end{array}$ & $\begin{array}{c}28 \\
100 \%\end{array}$ & $\begin{array}{c}0 \\
0 \%\end{array}$ & \multirow{3}{*}{$\begin{array}{c}83,3 \\
\text { Alta } \\
\text { presencia }\end{array}$} \\
\hline $\begin{array}{l}\text { 2. ¿Se planifica el tiempo que estará el sistema fuera de } \\
\text { operación? }\end{array}$ & $\begin{array}{c}28 \\
100 \%\end{array}$ & $\begin{array}{c}0 \\
0 \%\end{array}$ & \\
\hline $\begin{array}{l}\text { 3. ¿Corrigen las fallas que se presentan en el momento } \\
\text { específico? }\end{array}$ & $\begin{array}{c}14 \\
50 \%\end{array}$ & $\begin{array}{c}14 \\
50 \%\end{array}$ & \\
\hline \multicolumn{4}{|l|}{ Indicador: Proactivo } \\
\hline $\begin{array}{l}\text { 4. ¿Se realizan actividades de mantenimiento que } \\
\text { permiten incrementar la vida útil de los equipos } \\
\text { mediante un control de la falla? }\end{array}$ & $\begin{array}{c}17 \\
60,7 \%\end{array}$ & $\begin{array}{c}11 \\
39,3 \%\end{array}$ & $\begin{array}{l}79,8 \% \\
\text { Alta } \\
\text { presencia }\end{array}$ \\
\hline $\begin{array}{l}\text { ¿Se establece un control para cada una de las máquinas } \\
\text { que requiere mantenimiento? }\end{array}$ & $\begin{array}{c}28 \\
100 \%\end{array}$ & $\begin{array}{c}0 \\
0 \%\end{array}$ & \\
\hline $\begin{array}{l}\text { ¿Se fortalecen los procesos mediante análisis de modos } \\
\text { de fallas? }\end{array}$ & $\begin{array}{c}22 \\
78,6 \%\end{array}$ & $\begin{array}{c}6 \\
21,4 \%\end{array}$ & \\
\hline \multicolumn{4}{|c|}{ PROMEDIO: 87,62 ALTA PRESENCIA } \\
\hline
\end{tabular}

Al detalle se aprecia que todos los tipos de mantenimiento, considerados en esta investigación: predictivo, preventivo, programado, correctivo y proactivo, arribaron a la categoría de alta presencia. No obstante, tanto en el correctivo y como en el proactivo se observan dos actividades ubicadas en la categoría mediana presencia, lo cual es indicativo de oportunidades de mejora a este respecto.

Estos resultados logran validar a Duffuaa y otros (2010), para quienes los tipos de mantenimiento han evolucionado con el tiempo, actualmente existen variados sistemas que pueden presentarse en las instalaciones de operación, que exigen diferentes modos de mantenimiento para preservar la continuidad de los procesos productivos y sustentar la rentabilidad operacional, entre los cuales se encuentran: predictivo, preventivo, programado, correctivo y proactivo. También alcanzan congruencia con el criterio de la investigadora, para quien estos cinco tipos de mantenimiento son los de mayor pertinencia y aplicabilidad dentro del sector.

Ahora bien, al detalle de cada uno de los indicadores utilizados para medir la dimensión se puede apreciar que en el tipo de mantenimiento predictivo los encuestados atribuyeron alta presencia a estos procesos, con un $100 \%$ en la opción si para todos los ítems involucrados; de manera que al preguntarles, si se evalúan las máquinas antes de iniciar una actividad vinculada en las planteas compresoras de gas, si la empresa cuenta con un historial de todos los equipos que requieren mantenimiento, y si se analizan las condiciones de los equipos mientras se encuentren en funcionamiento, para todos los encuestados si se realiza.

Los resultados constatan que las actividades correspondientes al mantenimiento predictivo poseen alta presencia dentro de la gestión de mantenimiento, por ello, se deduce que hay alta congruencia con lo expuesto por Nava (2010), para quien este tipo de 
mantenimiento se basa en predecir la falla antes de que ésta se produzca. Se trata de adelantarse al momento en que el equipo deja de trabajar en sus condiciones óptimas.

Para lograrlo se utilizan herramientas y técnicas de monitores de parámetros físicos. Involucra actividades que se desarrollan para detectar cualquier anomalía en equipos en funcionamiento, aplicando la tecnología para la interpretación de datos obtenidos a través de un diagnóstico, con instrumentos colocados en diferentes partes del equipo o bien sea por toma de muestras.

De igual manera, alcanzan en gran medida a validar lo establecido por la investigadora, cuando afirma que este tipo de mantenimiento consiste en reemplazar o reparar partes, piezas, componentes 0 elementos justo antes que empiecen a fallar o a dañarse. En el programa de este tipo de mantenimiento se analizan las condiciones del equipo mientras este se encuentra funcionando o en operación, permitiendo de esta manera ajustar las operaciones y su periodicidad a un máximo de eficiencia. Esto es siempre menos costoso y confiable que el intervalo de mantenimiento preventivo, basado en factores como las horas máquina o alguna fecha prefijada

En la tabla 1 se muestran las respuestas correspondientes al indicador mantenimiento preventivo. Se aprecia que para el $96,4 \%$ de los encuestados en las plantas de gas bajo estudio, se dan con alta presencia las actividades 0 procesos relacionados al indicador. De tal forma, se tiene alta presencia, con el $100 \%$ de respuestas afirmativas, de que se ejecuten actividades para determinar la condición real de las máquinas examinadas, y analicen las condiciones de los equipos mientras se encuentran funcionando. Al mismo tiempo que para el $89,3 \%$, la empresa cuenta con un historial del mantenimiento realizado a todos los equipos.

Así, se logra alta congruencia con Duffuaa y otros (2010), cuando afirman que el mantenimiento preventivo, se da antes de que ocurra la falla o avería y se efectúa en condiciones controladas, sin la existencia de algún error en el sistema. Se realiza a razón de la experiencia, pericia del personal a cargo, los cuales son los encargados de determinar el momento necesario para llevar a cabo dicho procedimiento; también se puede estipular el momento adecuado a través de manuales técnicos.

A juicio de la investigadora, dado que se tiene alta presencia de este tipo de mantenimiento, en estas plantas se están aprovechando las oportunidades que ofrece el mismo, lo cual involucra utilizar todos los medios disponibles incluso los estadísticos, para determinar la frecuencia de las inspecciones, revisiones, sustitución de piezas claves, probabilidad de aparición de averías, vida útil, u otras. Así entonces, consiste en programar revisiones de equipos, apoyándose en la experiencia e históricos obtenidos de las máquinas y equipos.

De igual manera, en la tabla 1 se presenta la frecuencia relativa de las respuestas correspondientes a los ítems 7 , 8 y 9 pertenecientes al tipo de mantenimiento programado, indicando que en las plantas estudiadas se realiza con alta presencia el mismo, arribando este indicador a una frecuencia de $78,6 \%$ de respuestas afirmativas. Ha de notarse que todas las actividades medidas para este indicador, alcanzan la categoría de alta presencia con concentración de respuestas afirmativas al mismo nivel que el indicador $78,6 \%$. Lo cual indica que en las plantas 
analizadas se establecen controles destinados a detectar fallas potenciales, analizan las condiciones de los equipos mientras se encuentran funcionando, $\mathrm{y}$ programan las actividades para llevar a cabo un desempeño productivo efectivo.

Estos resultados, al reflejar alta presencia, ponen de manifiesto alta coincidencia con lo expresado por González (2005), quien afirma que el mantenimiento programado es el que se efectúa a intervalos predeterminados de tiempo, número de operaciones, recorridos, y otros. Consiste en establecer controles para detectar fallos potenciales, servicios programados y revisiones planificadas, más la sustitución de las piezas averiadas o con tendencia a la avería. Equivale al término mantenimiento rutinario, planificado o sistemático. Presta mucha atención a las recomendaciones del fabricante y otras personas conocedoras del tema, para obtener un cronograma de aplicación.

A criterio de la investigadora, esta situación mostrada evidencia que en las plantas de gas se presta el debido valor al mantenimiento programado, considerándolo como el conjunto de distintas actividades programadas con el fin de llevar a cabo un desempeño productivo para la máquina. Por lo cual, este surge como el resultado de la dedicación del departamento de mantenimiento, teniendo como objetivo ajustar la programación del equipo para desarrollar las tareas en el momento menos perjudicial para la producción.

En lo concerniente al tipo de mantenimiento correctivo, los resultados demuestran alta presencia del mismo en la gestión que se analiza, ostentando un 83,3\% (promedio) de respuestas en la opción afirmativa si (tabla 1). Así, los encuestados respondieron en un $100 \%$ en la opción si al consultárseles: si realizan actividades correctivas cuando ocurre una falla, y planifican el tiempo que estará el sistema fuera de operación. No obstante, solo un $50 \%$ respondió de forma afirmativa que se corrigen las fallas que se presentan en el momento específico, indicando mediana presencia de esta actividad.

Situación ésta que valida lo postulado por Nava (2010), para quien el tipo correctivo es aquel trabajo que involucra una cantidad determinada de tareas de reparación no programadas con el objetivo de restaurar la función de un activo una vez producido un paro imprevisto (parada forzada). También validan a Zambrano y Leal (2007), cuando afirman que las causas que pueden originar un paro imprevisto se deben a desperfectos no detectados durante las inspecciones predictivas.

Asimismo, validan a la investigadora quien opina que el correctivo no se puede eliminar en su totalidad, por lo tanto, una gestión correcta extraerá conclusiones de cada parada e intentará realizar la reparación de manera definitiva, ya sea en el mismo momento o programando un paro, para que esa falla no se repita. Por lo cual es importante tener en cuenta en el análisis de la política de mantenimiento a implementar, que en algunas máquinas o instalaciones el correctivo sería el sistema más rentable.

En último caso, para la dimensión estudiada, se muestra lo obtenido para el tipo de mantenimiento proactivo, el cual, tal como se ve en la tabla 1, arribó a alta presencia con una concentración de respuestas afirmativas del $79,8 \%$ en promedio. Detallando se tiene: alta presencia con el $100 \%$ de las respuestas afirmativas de que establecen un control 
para cada una de las máquinas que requiere mantenimiento; el 78,6\% afirma que se fortalecen los procesos mediante análisis de modos de fallas, otorgándole también alta presencia; mientras que para el $60,7 \%$ se realizan actividades de mantenimiento que permiten incrementar la vida útil de los equipos mediante un control de la falla, indicando mediana presencia de esta actividad.

Estos resultados evidencian alta congruencia con García (2009), quien señala al tipo proactivo como una gran cantidad de técnicas para alargar la disponibilidad de las máquinas. Considera que la idea principal de un mantenimiento proactivo es el análisis de las causas raíz de las fallas, así estas causas se pueden remediar y los mecanismos de falla que se puedan eliminar gradualmente, mejoraran la operación de las máquinas. Visto así, el tipo proactivo no se encarga de corregir errores o defectos de las máquinas si no en lo que los causa. Lo que implicaría uno de los mantenimientos más baratos.

Asimismo, se valida a la investigadora cuando afirma que, es importante considerar que la productividad aumentará en la medida en que las fallas de las máquinas disminuyan de una forma sustentable en el tiempo, para lograrlo, resulta indispensable contar con la estrategia de mantenimiento más apropiada y con personal capacitado tanto en el uso de las técnicas de análisis como en el diagnóstico de fallas, con conocimiento suficiente sobre las características de diseño y funcionamiento de las máquinas.

A manera de resumen de esta dimensión, se describieron los tipos de mantenimiento utilizados con mayor frecuencia hoy en día, observándose que en las plantas compresoras de gas en la industria petrolera venezolana, Distrito Lagunillas, se posee alta presencia de todos los tipos de mantenimiento, acotándose que la elección del tipo dependerá en gran medida de las estrategias planteadas en la planificación del mantenimiento. Aun cuando, se resalta, que tanto en el mantenimiento correctivo como en el proactivo se detectó una actividad que ofrece oportunidades de mejora.

En detalle, en el mantenimiento correctivo se catalogó con mediana presencia el que se corrijan las fallas que se presentan en el momento específico; y en el proactivo, con mediana presencia también, el que se realizan actividades de mantenimiento que permiten incrementar la vida útil de los equipos mediante un control de la falla.

\section{CONCLUSIONES}

En lo que concierne al objetivo, donde se describieron los tipos de mantenimiento aplicados en las plantas compresoras de gas en la industria petrolera venezolana, Distrito Lagunillas, se concluye que estas plantas otorgan alta presencia a los cinco tipos de mantenimiento estudiados en la gestión de mantenimiento que llevan a cabo: predictivo, preventivo, programado, correctivo y proactivo.

Es necesario destacar que si bien se obtuvo alta presencia de estos tipos de mantenimiento en la gestión analizada, se detectaron oportunidades de mejora en las actividades medidas para los mantenimientos programado, correctivo y proactivo, lo cual permite a la investigadora sugerir la necesidad de fortalecer el cumplimiento de cada uno de los tipos de mantenimiento analizados. 
REFERENCIAS

Altmann, C. (2005). El análisis de aceite como herramienta del mantenimiento proactivo en flotas de maquinaria pesada. 1er Congreso Uruguayo de Mantenimiento, Gestión de Activos y Confiabilidad. Montevideo, Uruguay

Duffuaa, S., Raouf, A. y Campbell, J. (2010). Sistemas de mantenimiento. Planificación y control. Editorial Limusa. México

García, R. (2009). Mantenimiento Industrial: Manual de operación y administración. Segunda edición. Editorial Trillas. Ciudad de México. México

González, F. (2005). Teoría y práctica del mantenimiento industrial avanzado. Segunda edición. Editorial Fundación Confemetal. Príncipe de Vegara, Madrid. España

Jiménez, K. y Milano, T. (2006). Planificación y gestión del mantenimiento industrial. Un enfoque estratégico y operativo. Editorial Panapo. Caracas. Venezuela

Martínez, L. (2007). Organización y planificación de sistemas de mantenimiento. Centro de altos estudios gerenciales. Instituto Superior de Investigación y Desarrollo. Segunda Edición. Caracas. Venezuela

Nava, J. (2010). Aplicación práctica de la teoría de mantenimiento. 2 da edición. Edit. Consejo de publicaciones de la Universidad de los Andes. Mérida, Venezuela.

Newbrough, C. (2010). Mantenimiento. Ejecución y control. Alfaomega Grupo Editor, S.A. México D.F., México
Norma Covenin 3049-93

Mantenimiento, Definiciones. Elaborada por el comité Técnico de Normalización CT-3: Construcción, aprobada por COVENIN en su reunión No. 124 de fecha 93-12-01 Venezuela

Perdomo, M. (2007). Modelo de gestión de mantenimiento: una visión estratégica. II Congreso de Ingeniería Mecánica ASME- Perú

Rodríguez, J. (2008). Gestión de mantenimiento. Introducción a la teoría del mantenimiento. Casos empresariales. Consultado el 20/03/2020. Disponible en http://www.scribd.com/doc/7497765/ gestion-del-mantenimiento

Salih, H. (2010). Ingeniería del mantenimiento industrial y servicios integrales de lubricación. Edit. T.B.N. Islas Canarias, España

Segura, A. (2005). Gestión de procesos. Desde la mejora hasta el rediseño. 2 da ed. Edit. Editorial Evolución S.A. Santiago de Chile, Chile

Torres, N. (2006). Como realizar mantenimiento. Editorial Norma. Caracas. Venezuela

Trashorras, J. (2006). Desarrollo de instalaciones electrónicas en los edificios. Cuarta edición, segunda reimpresión. Ediciones Paraninfo, S.A. Madrid. España

Venahin, C. (2008). Sistemas de mantenimiento planificación integral. Edit. Limusa. México D.F., México

Zambrano, S. y Leal, S. (2007). Fundamentos Básicos de Mantenimiento. Segunda edición. Fondo Editorial UNET. Táchira. Venezuela 